\title{
Incidence and predictors of adverse pregnancy outcomes with maternal HIV-1 immune reconstitution inflammatory response syndrome among women within reproductive age in selected hospitals, Nairobi, Kenya (A prospective cohort study)
}

John Kyalo Muthuka ( $\boldsymbol{\sim}$ johnmuthuka@gmail.com )

Kenya Medical Training College

Yeri Kombe

Kenya Medical Research Institute

Anselimo Makokha

Jomo Kenyatta University of Agriculture and Technology

Michael Kiptoo

Kenya Medical Training College

Research article

Keywords: maternal HIV-1 immune reconstitution inflammatory response syndrome, maternal HIV-1 immune reconstitution inflammatory response syndrome, prevention of mother to child transmission (PMTCT)

Posted Date: October 6th, 2020

DOl: https://doi.org/10.21203/rs.3.rs-85433/v1

License: (1) This work is licensed under a Creative Commons Attribution 4.0 International License. Read Full License 


\section{Abstract}

Background: With enhanced rollout of anti-retroviral therapy (ART) following prevention of mother to child transmission (PMTCT) and the associated burden of opportunistic infections in developing countries, human immunodeficiency virus-immune reconstitution inflammatory syndrome (HIV-IRIS) may remain a public health area of major concern especially, in pregnancy. Ascertaining the association between maternal HIV infection and adverse maternal and birth related outcomes could be conflicted by the relationship between maternal-HIV- IRIS and the adverse pregnancy-fetal outcomes (APFOs) in HIVinfected mothers. We sought to estimate the incidence and determine possible predictors for adverse pregnancy-fetal outcomes with maternal-HIV-IRIS in ART naïve HIV infected pregnant women.

Methodology: Subjects grouped in to IRIS exposed and non-IRIS exposed were followed from the end of first trimester for six and half months. Chi-square test was used to establish the association between the variables at $p$-value $<0.05$. Regression analysis was performed to identify independent predictors of APFOs. Adjusted Relative Risk at 95\% confidence interval was determined.

Results: The IRIS exposed pregnant women, had a $26.47 \%$ adverse pregnancy-fetal outcomes cumulative incidence compared to $10.78 \%$ among IRIS non-exposed women. The APFO incidence rate estimate was 0.012 and 0.0045 per person's week respectively. IRIS cases had 2.46 times the risk of experiencing an APFO compared to those who did not [OR=3; 95\% Cl: 1.4-6.4; $\mathrm{P}=.004]$, at bivariate analysis, not sustained at the multivariate analysis [AOR=1.6; $95 \% \mathrm{Cl}: 0.4-5.8 ; \mathrm{P}=.508$ ]. Multiple logistic regression dropped maternal HIV-IRIS and revealed; HIV-RNA viral load at baseline of above $50 \mathrm{copies} / \mathrm{ml}$ [AOR=2.7; 95\%Cl: 1.2-6.3; $P=.017$, Maternal placental syndrome(MPS) characterized by hypertensive event [AOR=0.1; $95 \% \mathrm{Cl}: 0.0-1.0 ; \mathrm{P}=.052]$ and mother's general health during delivery [AOR= 4; 95\% $\mathrm{Cl}: 4.0: 1.8-9.1 ; \mathrm{P}=.001]$ as independent predictors of APFOs.

Conclusion: There is a higher incidence of APFOs among maternal HIV-IRIS diagnosed pregnant women as compared to non-HIV maternal IRIS diagnosed pregnant women. In particular, MPS characterized by an hypertensive event, HIV-RNA viral load at baseline of above 50 copies/ $\mathrm{ml}$ and mother's general health during delivery are key predictors of an adverse pregnancy outcome. Interventions to reduce the associated risk predictors identified in this analysis should be studied for their effects on reducing APFOs.

\section{Background}

Immune Reconstitution Inflammatory Response Syndrome is an immune recovery disorder referring to pathogen-specific inflammatory responses in HIV after starting or re-initiation of ARV treatment or shift to active ARV regimen. IRIS is usually associated with an increase in CD4 cell count and/or a rapid decrease in HIV viral load. The time of presentation is usually within the first 4 to 8 weeks after initiation of ARV therapy; however, it has occurred weeks after starting and in sequestered sites (1). HIV-infected individuals are at high risk of developing numerous opportunistic infections. The severity of these 
infections may increase proportional to the immunosuppressant degree (3). This describes two distinct outcomes; an exacerbation of a partially or completely treated opportunistic diseases, paradoxical IRIS and an inflammatory response to a previously un-diagnosed, often more pronounced than the typical presentation of this Ol, the unmasking IRIS (5). A report by WHO \& KDHS, 2009 showed that, HIV-infection among women is generally higher than men as per the latest finding in Africa based on several factors and this translates to pregnant women at large. This is associated with negative neonatal outcomes such as neonatal mortality among women by a study done in Kenya (4).

The overall incidence of IRIS is unknown, but is dependent on the population studied and its underlying opportunistic infections burden. The infectious pathogens most frequently associated with the syndrome have been documented. No specific treatment option exists and depends on the underlying infectious agent and its clinical presentation. A variety of mycobacterium, viral, fungal and parasitic opportunistic infections is linked with immune reconstitution inflammatory response syndrome (2). Mortality related with IRIS is uncommon; however, associated high morbidity contributes to burden on the health-care system (6). This has become a public health concern, as ART use has been associated with increased IRIS inform of opportunistic conditions and other non-infectious conditions. A few autoimmune and other non-infectious conditions may worsen or appear after HAART is begun; suggesting that inflammation induced by an IRIS-like syndrome is responsible. Whether such relationship represents a casual or a coincidental finding, this is unproven at present (7).

The clinical outlook of each individual case of IRIS is variable and data from trials have not been published thus far to give sound guidelines. Recommendations are based on clinical experience, case reports, case series, and other expert opinion (8). To diagnose IRIS, presence of a new Ol or other illness, failure of HIV treatment and failure of treatment for a known OI (e.g., owing to drug resistance, inadequate treatment, or poor adherence) must be ruled out. The severity of IRIS varies widely, from mild to life-threatening outcomes. Treatment varies according to the specific pathogen and clinical situation, but typically includes continuing ART if possible, treating the OI as indicated, and adding antiinflammatory therapy (including corticosteroids) as needed (9).

The presentation is largely a clinical diagnosis, and other conditions must be eliminated, as indicated above. To consider IRIS in the differential diagnosis, clinicians must recognize the clinical findings (typical or atypical) of a specific Ol and the temporal association with treatment (usually after ART initiation, but IRIS may occur with treatment of the OI alone). For example, for a patient with TB who has recently initiated ART after responding to treatment of TB, the "red flags" for a diagnosis of IRIS (rather than progression of the TB) would include new or worsening fever, new effusions, and new or worsening lymphadenopathy, in the absence of poor adherence to TB treatment or drug-resistant TB (11).

In HIV-infected pregnant women, the administration of ART during pregnancy and/or intra-partum significantly reduces the risk of mother-to-child transmission (MTCT) of HIV (12). Although the beneficial effects of antiretroviral (ARV) therapy for preventing mother-to-child transmission are indisputable, studies in developed and developing countries have reported conflicting findings on the association 
between ARV exposure and adverse birth outcomes. ART also has directly and indirectly been found to be of significant contribution to poor pregnancy outcomes (13). Also, subgroup of patients experiences a clinical deterioration as a consequence of rapid and dis-regulated restoration of antigen specific immune responses during the treatment (14).

Given that the evidence for the incidence of up to 30 percent of ART responders developing one or more inflammatory syndromes consistent with IRIS (15), the conditions associated with it will have their greatest impact in resource-poor countries, where patients are often very immune-deficient, including pregnancy associated immune-depression and opportunistic pathogens when therapy is commenced (10).

In the present study, the cumulative incidence and incidence rate was estimated and the independent predictors for adverse pregnancy-fetal outcomes among ART naïve pregnant women of reproductive age were determined.

\section{Methods}

The present study was conducted at Kenyatta National and Mbagathi Hospitals both located in Nairobi County. The subjects were recruited and followed from the end of first trimester for six and half months after they were confirmed to be HIV positive, and put on ARV treatment with a defined case of HIV-IRIS as exposed cohort and non-HIV - IRIS as the non- exposed cohort, immediately after the first trimester.

A total of 204 subjects of both cohorts were included in the final analysis following subsequent elimination process at an equal ratio to the unexposed cohort (1:1). A pretested data questionnaire abstraction tool was used. Matching by age and parity among pregnant women infected with HIV and confirmed of the status by test at least in the first trimester was performed. These protocols were designed to describe the characteristics of enrolled pregnant women, use of ARV regimens, and adverse pregnancy-fetal outcomes.

The pregnant women were followed after confirmation of IRIS exposure status and meeting the inclusion criteria, until postpartum through delivery stage. Pre testing of the abstraction tool was done by the principal investigator and the research assistants. The information abstraction tool was uniform for both groups with coded values to conceal identity, majorly focusing on the primary outcome variable with link to maternal HIV immune reconstitution inflammatory syndrome response status and potential confounders.

The data collected was analyzed using SPSS version 25.0. Data were summarized in tables and figures. Continuous variables were presented as the mean \pm standard deviations (SDs). Chi-square test was used to establish the association between the dependent and independent variables and the level of statistical significance was set at $p$-value $<0.05$. Multiple logistic regression analyses were performed to adjust for confounding. Adjusted Odds ratio (AOR) with corresponding 95\% confidence interval was estimated. The 
approval to carry on with the research was sought from $\mathrm{KNH} / \mathrm{UON}$ - ERC and permission for the selected facilities' entry and data collection was sought accordingly.

\section{Results}

\section{Social-demographic and economic characteristics of HIV-positive ART initiated pregnant women}

Data was gathered from a total of 204 HIV-positive ART initiated pregnant women, that is, 102 diagnosed with maternal HIV immune reconstitution inflammatory response syndrome and 102 without the diagnoses of the syndrome seeking antenatal care services from Mbagathi and Kenyatta National Hospitals in Nairobi County. Most, 103 (50.5\%) HIV positive ART initiated women were aged between 2039 years. Majority, 163 (79.9\%) were from Nairobi region while the rest from other counties, with most, $131(64.2 \%)$ of them being married women. Over 90\%, 189 (92.6\%) of HIV positive ART initiated women were Christians with only 15 (7.4\%) being Muslims. Majority of HIV positive ART initiated 109 (53.4\%) had secondary education. Only $49(24.0 \%)$ had tertiary education. Almost half of them, 101 (49.5\%) were self employed and about a similar proportion of them $86(42.2 \%)$ were unemployed. Only $16(7.8 \%)$ were in civil service. On income source, 46 (22.5\%) were housewives while the majority, 142 (69.6\%) had some work to generate some income. Only $16(7.8 \%)$ were not known of their income source status. This generally depicted that, on income generation, most of these pregnant women are not employed and most income is self employment (Table 1). 
Table 1

Social-demographic and economic characteristics

\begin{tabular}{|ll|}
\hline Variable & Frequency (\%) \\
\hline Maternal age Category in years & $73(35.8)$ \\
$30-29$ & $103(50.5)$ \\
$40-49$ & $28(13.7)$ \\
\hline Location/address & \\
Nairobi & $163(79.9)$ \\
Outside Nairobi & $41(20.1)$ \\
\hline Education level & \\
No formal education & $21(10.3)$ \\
Primary & $24(11.8)$ \\
Secondary & $109(53.4)$ \\
Higher/university & $49(24.0)$ \\
Not recorded & $1(0.5)$ \\
\hline Occupation & \\
Unemployed & $86(42.2)$ \\
Civil servant & $16(7.8)$ \\
Self-employed & $101(49.5)$ \\
Not recorded & $1(0.5)$ \\
\hline Religion & $189(92.6)$ \\
Christian & $15(7.4)$ \\
Muslim & $131(64.5)$ \\
\hline Marital status & \\
Single & \\
Married & \\
\hline Not recorded & \\
\hline
\end{tabular}




\begin{tabular}{|ll|}
\hline Variable & Frequency (\%) \\
\hline Income source & $142(69.6)$ \\
Working( Self employed or employed) & $46(22.5)$ \\
House wife & $16(7.8)$ \\
Not recorded & \\
\hline
\end{tabular}

\section{Socio-demographic characteristics of women experiencing APFOs and not experiencing APFOs}

A total of 204 women were included in the final analysis where 102 who fulfilled the criteria for IRIS diagnosis as per experts' opinions within the first trimester represented as exposed and 102 as an unexposed within the first trimester as the comparator arm, following subsequent elimination from the exposed arm from 133 IRIS indentified initially. Of these 204 women, 38 (18.62\%) experienced an adverse pregnancy outcome (of a particular nature). Table 4.1 shows some of the selected socio-demographic characteristics of women with and without APFOs. The common maternal age among the women was between 30-39 years with larger proportion of this age category being in women who did not experience adverse pregnancy-fetal outcomes but this observation was not significant 19 (18.4) ; 84 (81.6) [OR = 0.9; $95 \% \mathrm{Cl}: 0.7-2.4 ; \mathrm{P}=.728]$. Mothers' status of being separated as regards marital status was found to be slightly significant for experiencing an adverse pregnancy outcome. Adverse pregnancy-fetal outcomes were more likely among mothers with separated marital status over four times compared to women without adverse pregnancy-fetal outcomes $\mathrm{OR}=4.2 ; 95 \% \mathrm{Cl}: 1.0-16.9 ; \mathrm{P}=0.044]$. There was a difference in the proportion of woman's level of education among women experiencing adverse pregnancy-fetal outcomes and those not experiencing but this observation was not significant $(P>0.95)$. In addition, there was no statistically significant observation at $(P>0.05)$ between the other women's socio-demographic characteristics and adverse pregnancy outcome as indicated in (Table 2). 
Table 2

Comparison of Social-demographic characteristics between women who experienced adverse pregnancy-fetal outcomes and those who did not

\begin{tabular}{|c|c|c|c|c|}
\hline \multirow[t]{3}{*}{ Variable } & \multicolumn{2}{|l|}{ APFO } & \multirow[t]{3}{*}{ OR $(95 \% \mathrm{Cl})$} & \multirow[t]{3}{*}{$P$ value } \\
\hline & Yes & No & & \\
\hline & n (\%) & $\mathrm{n}(\%)$ & & \\
\hline Maternal age in years & $15(20.5)$ & $58(79.5)$ & 1.0 & 0.728 \\
\hline $20-29$ & $19(18.4)$ & $84(81.6)$ & $0.9(0.4-1.7)$ & 0.473 \\
\hline $30-39$ & $4(14.3)$ & $24(85.7)$ & $0.6(0.2-2.1)$ & \\
\hline \multicolumn{5}{|l|}{$40-49$} \\
\hline Location/address & $29(17.8)$ & $134(82.2)$ & $0.8(0.3-1.8)$ & 0.541 \\
\hline Nairobi & $9(22.0)$ & $32(78.0)$ & 1.0 & \\
\hline \multicolumn{5}{|l|}{ Outside Nairobi } \\
\hline Education level & $1(4.8)$ & $20(95.2)$ & 1.0 & 0.233 \\
\hline No formal education & $4(16.7)$ & $20(83.3)$ & $4.0(0.4-39.0)$ & 0.123 \\
\hline Primary & $22(20.2)$ & $87(79.8)$ & $5.1(0.6-39.8)$ & 0.104 \\
\hline Secondary & $11(22.4)$ & 38 (77.6) & $5.8(0.7-48.1)$ & \\
\hline \multicolumn{5}{|l|}{ Higher/university } \\
\hline Occupation & $15(17.4)$ & $71(82.6)$ & 1.0 & 0.479 \\
\hline Unemployed & $4(25.0)$ & $12(75.0)$ & $1.6(0.4-5.6)$ & 0.946 \\
\hline Civil servant & $18(17.8)$ & $83(82.2)$ & $1.0(0.5-2.2)$ & \\
\hline \multicolumn{5}{|l|}{ Self-employed } \\
\hline Religion & $36(19.0)$ & $153(81.0)$ & $1.5(0.3-7.1)$ & 0.742 \\
\hline Christian & $2(13.3)$ & $13(86.7)$ & 1.0 & \\
\hline \multicolumn{5}{|l|}{ Muslim } \\
\hline Marital status & $11(18.3)$ & 49 (81.7) & $1.2(0.5-2.6)$ & 0.693 \\
\hline Single & $21(16.0)$ & $110(84.0)$ & 1.0 & 0.044 \\
\hline Married & $4(44.4)$ & $5(55.6)$ & $4.2(1.0-16.9)$ & 1.000 \\
\hline Separated & 0 & $1(100)$ & 0 & \\
\hline Windowed & & & & \\
\hline
\end{tabular}




\begin{tabular}{|lllll|}
\hline Variable & \multicolumn{2}{l}{ APFO } & OR (95\% Cl) & P value \\
\cline { 2 - 5 } & Yes & No & & \\
& $\mathbf{n}(\%)$ & $\mathbf{n}(\%)$ & & \\
\hline Income source & $3(25.0)$ & $9(75.0)$ & $1.4(0.3-6.1)$ & 0.680 \\
Employed & $25(19.2)$ & $105(80.8)$ & $1.0(0.4-2.3)$ & 0.961 \\
Self employed & $9(19.6)$ & $37(80.4)$ & 1.0 & \\
House wife & & & & \\
\hline
\end{tabular}

Parity distribution among all women $(n=204)$

Majority of women $(47.1 \%)$ had a parity of $2-3$ followed closely by women who had a parity of 1 at $43.1 \%$. Women with a parity of $6-7$ were only $1 \%$ of the total (Fig. 1 )

Proportion of women experiencing APFOs compared to women not experiencing APFOs

Out of 102 women exposed to IRIS, 27 experienced adverse pregnancy-fetal outcomes compared to 11 among 102 women not exposed to IRIS (Fig. 2)

Incidence of adverse pregnancy-fetal outcomes in women experiencing maternal HIV -IRIS compared to women not experiencing maternal HIV -immune reconstitution inflammatory response syndrome over the entire follow-up period (six and half months post IRIS identification:

Out of 102 IRIS exposed women, $26.47 \%$ experienced adverse pregnancy-fetal outcomes compared to $10.78 \%$ among 102 IRIS non-exposed women. The cumulative incidence of adverse pregnancy-fetal outcomes in IRIS exposed group was therefore over double compared to that of the IRIS unexposed (comparator) group. The relative risk of experiencing an adverse pregnant outcome was $26.47 / 10.78=$ 2.46. This suggests that women with IRIS had 2.46 times the risk of experiencing adverse pregnancy outcome compared to those who did not. Exposure to IRIS contributed to adverse pregnancy-fetal outcomes [OR = 3; 95\%Cl: 1.4-6.4; P = .004] (Table 3). 
Table 3

A contingency of Cumulative incident and RR of adverse

pregnancy outcome in women with maternal HIV -immune reconstitution inflammatory response syndrome as compared to

women without maternal HIV -immune reconstitution inflammatory response syndrome

\begin{tabular}{|lllll|}
\hline Variable & APFO & & OR (95\% Cl) & P value \\
\cline { 2 - 3 } & Yes & No & & \\
& $\mathbf{n}(\%)$ & $\mathbf{n}(\%)$ & & \\
\hline IRIS & $27(26.5)$ & $75(73.5)$ & $3.0(1.4-6.4)$ & 0.004 \\
Yes & $11(10.8)$ & $91(89.2)$ & 1.0 & \\
No & & & & \\
\hline
\end{tabular}

Incidence rate estimate: The subjects were followed for duration of six and half months (26weeks) post IRIS identification and allocation, with two week post-partum period included as the last phase of followup. There were visits: at the end of second trimester, that is at sixth month during delivery /post partum periods with spacing of three months each visit. Since the exact time at risk could not be determined easily, each subject was allocated $50 \%$ of the duration of time at risk of adverse pregnancy outcome among the two cohorts. At the first and the last visits, the IRIS exposed and IRIS non-exposed women had the following adverse pregnancy-fetal outcomes each;

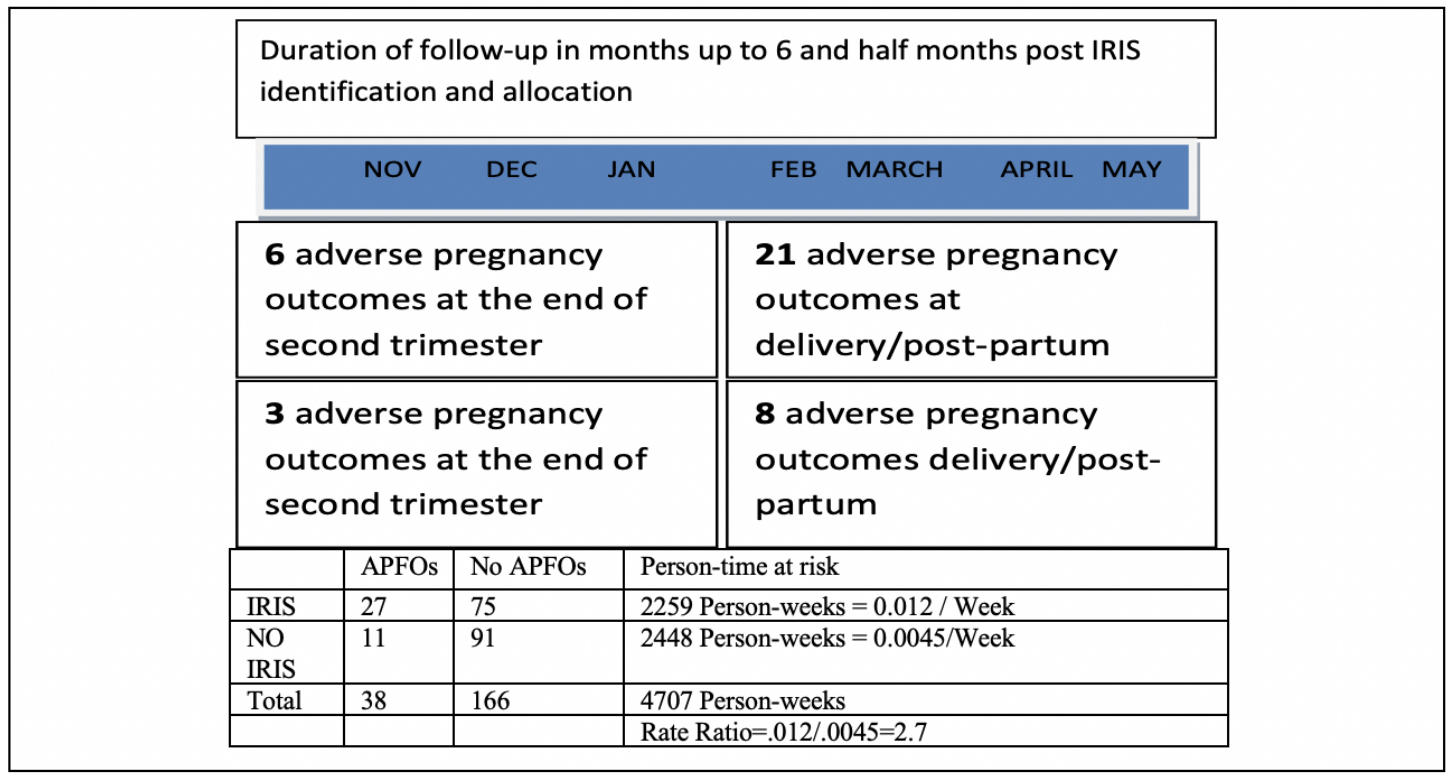

Adverse pregnancy-fetal outcomes and subjects' enrollment baseline clinical and immunological characteristics: Analysis summery of baseline clinical characteristics with adverse pregnancy-fetal outcomes. 
There was an increase in proportion (incidence) of adverse pregnancy-fetal outcomes among women with IRIS from the contingency table (Table 2) (26.5\%) compared to the women without IRIS (10.8\%), [OR $=3.0 ; 95 \% \mathrm{Cl}$ : $1.4-6.4 ; \mathrm{P}=.004]$. Adverse pregnancy outcome incidence was significantly more among women with opportunistic infections (27.8\%) when compared to the women without opportunistic infections $[\mathrm{OR}=2.3 ; 95 \% \mathrm{Cl}: 1.1-4.8 ; \mathrm{P}=.013]$. The incidence of adverse pregnancy-fetal outcomes was slightly significantly associated with the cluster of differentiation (CD4 Count) of 350 cells per cubic millimeter and below at (23.6\%) higher than women who had cluster of differentiation (CD4 Count) of 350 cells per cubic millimeter and above at $(13.6 \%)$ [OR $=2.1 ; 95 \% \mathrm{Cl}: 1.0-4.4 ; \mathrm{P}=.051]$. On the contrary, it was found that, HIV ribonucleic acid viral loads level of 50 copies $/ \mathrm{ml}$ and below was protective for adverse pregnancy-fetal outcomes 18 representing (31.0\%) as compared to 50 copies and above20 representing (13.7\%) [OR $=0.4 ; 95 \% \mathrm{Cl}: 0.2-0.7 ; \mathrm{P}=.004]$. Adverse pregnancy-fetal outcomes incidence was a bit higher $(25.0 \%)$ in women who experienced extensive skin physical examination compared to (17.4\%) women who did not experience extensive skin physical examination. However, there was no significant difference with respect to woman's HIV staging $(P>.05)$, previous medical history $(p=0.096)$, neurological extensive physical examination $(P=.392)$, full blood count $(p=.141)$ and basic blood chemistry $(p=1.000)$ among the women experiencing adverse pregnancy-fetal outcomes and women not experiencing adverse pregnancy-fetal outcomes (Table 4). 
Table 4

Comparison of women experiencing and not experiencing APFOs in relation subjects' enrollment baseline clinical characteristics

\begin{tabular}{|c|c|c|c|c|}
\hline \multirow[t]{3}{*}{ Variable } & \multicolumn{2}{|l|}{ APFO } & \multirow[t]{3}{*}{ OR $(95 \% \mathrm{Cl})$} & \multirow[t]{3}{*}{$P$ value } \\
\hline & Yes & No & & \\
\hline & n (\%) & n (\%) & & \\
\hline WHO-HIV Staging-2016 & $12(18.5)$ & $53(81.5)$ & 1.0 & - \\
\hline Primary stage & $17(15.2)$ & $95(84.8)$ & $0.8(0.4-1.8)$ & 0.570 \\
\hline Clinical stage 1 & 8 (33.3) & $16(66.7)$ & $2.2(0.8-6.3)$ & 0.141 \\
\hline Clinical stage 2 & $1(33.3)$ & $2(66.7)$ & $2.2(0.2-26.4)$ & 0.531 \\
\hline \multicolumn{5}{|l|}{ Clinical stage 3} \\
\hline Medical History & $8(32.0)$ & $17(68.0)$ & $2.3(0.9-5.9)$ & 0.096 \\
\hline Previous illnesses & $30(16.8)$ & $149(83.2)$ & 1.0 & 0.013 \\
\hline Yes & $20(27.8)$ & $52(72.2)$ & $2.3(1.1-4.8)$ & \\
\hline No & $18(13.6)$ & $114(86.4)$ & 1.0 & \\
\hline \multicolumn{5}{|l|}{ Opportunistic infections } \\
\hline \multicolumn{5}{|l|}{ Yes } \\
\hline \multicolumn{5}{|l|}{ No } \\
\hline Extensive Physical exam & $6(26.1)$ & $17(73.9)$ & $1.6(0.6-4.5)$ & 0.392 \\
\hline Neurological & $32(17.7)$ & $149(82.3)$ & 1.0 & 0.287 \\
\hline Yes & $9(25.0)$ & $27(75.0)$ & $1.6(0.7-3.7)$ & \\
\hline No & $29(17.4)$ & $138(82.6)$ & 1.0 & \\
\hline \multicolumn{5}{|l|}{ Skin } \\
\hline \multicolumn{5}{|l|}{ Yes } \\
\hline \multicolumn{5}{|l|}{ No } \\
\hline CD4 counts & $26(23.6)$ & $84(76.4)$ & $2.1(1.0-4.4)$ & 0.051 \\
\hline$<350$ & $12(12.9)$ & $81(87.1)$ & 1.0 & \\
\hline \multicolumn{5}{|l|}{$>350$} \\
\hline HIV viral loads & $20(13.7)$ & $126(86.3)$ & $0.4(0.2-0.7)$ & 0.004 \\
\hline > 50 copies $/ \mathrm{ml}$ & $18(31.0)$ & $40(69.0)$ & 1.0 & \\
\hline$<50$ copies/ml & & & & \\
\hline
\end{tabular}




\begin{tabular}{|c|c|c|c|c|}
\hline \multirow[t]{3}{*}{ Variable } & \multicolumn{2}{|l|}{ APFO } & \multirow[t]{3}{*}{ OR $(95 \% \mathrm{Cl})$} & \multirow[t]{3}{*}{$P$ value } \\
\hline & Yes & No & & \\
\hline & $\mathrm{n}(\%)$ & n (\%) & & \\
\hline Full Blood Count & $7(31.8)$ & $15(68.2)$ & $2.3(0.9-6.0)$ & 0.141 \\
\hline Abnormal & $31(17.0)$ & $151(83.0)$ & 1.0 & \\
\hline \multicolumn{5}{|l|}{ Normal } \\
\hline Basic blood Chemistry & $1(16.7)$ & $5(83.3)$ & $0.8(0.1-7.7)$ & 1.000 \\
\hline Abnormal & $37(18.7)$ & $161(81.3)$ & 1.0 & \\
\hline Normal & & & & \\
\hline
\end{tabular}

Comparison of women with adverse pregnancy-fetal outcomes and women without adverse pregnancyfetal outcomes by Maternal health (during pregnancy) and/or at birth and Type of ARV Combination:

In comparison, women with APFOs and women without APFOs by Maternal health (during pregnancy) and/or at birth and Type of ARV combination, it indicates that, a BMI value of $25.0-29.9 \mathrm{~kg} / \mathrm{m}^{2}$ (overweight) showed only and just certain trend toward significance $[\mathrm{OR}=2.1 ; 95 \% \mathrm{Cl}: 1.34-6.02 ; \mathrm{P}=$ 0.008 ] in relation to experiencing an adverse pregnancy outcome. On the contrary as it would be expected, the odds of adverse pregnancy-fetal outcomes in women with any maternal placental syndrome defining event (hypertensive disorder) was $80 \%$ less than in the women without maternal placental syndrome defining event with the true population effect between $100 \%$ and $10 \%$ [O.R $=0.2 ; 95 \% \mathrm{Cl}: 0.1-1 ; \mathrm{P}=0.031]$. This result was statistically significant that, maternal placental syndrome event (hypertensive disorders) was protective for adverse pregnancy-fetal outcomes although not fully conclusive as this was at the borderline. Prophylaxis during pregnancy, maternal substance abuse, maternal anemia, cesarean section delivery, obstetrical interventions, Rhesus factor, parity and any previous adverse infant outcome showed no significant association with experiencing an adverse birth outcome. Type of ART combination relative to adverse pregnancy-fetal outcomes was not significant and therefore no difference in the three clusters of combinations of the ART. All these with $\mathrm{P}>0.05$ (Table 5) 
Table 5

Comparison of women with and without APFOs by Maternal health and Type of ARV Combination

\begin{tabular}{|c|c|c|c|c|}
\hline \multirow[t]{3}{*}{ Variable } & \multicolumn{2}{|l|}{ APFO } & \multirow[t]{3}{*}{ OR (95\% Cl) } & \multirow{3}{*}{$\begin{array}{l}P \\
\text { value }\end{array}$} \\
\hline & Yes & No & & \\
\hline & $\mathrm{n}(\%)$ & $\mathrm{n}(\%)$ & & \\
\hline \multirow{3}{*}{$\begin{array}{l}\text { Prophylaxis during Pregnancy } \\
\text { Yes } \\
\text { No }\end{array}$} & \multirow{2}{*}{$\begin{array}{l}22 \\
(21.6)\end{array}$} & $80(78.4)$ & $1.5(0.7-3.0)$ & \multirow[t]{3}{*}{0.281} \\
\hline & \multirow{2}{*}{\multicolumn{2}{|c|}{$\begin{array}{l}16 \\
(15.7)\end{array}$}} & \multirow[t]{2}{*}{1.0} & \\
\hline & & & & \\
\hline \multirow{3}{*}{$\begin{array}{l}\text { Maternal Substance Abuse } \\
\text { Yes }\end{array}$} & $1(7.1)$ & $13(92.9)$ & \multirow{2}{*}{$\begin{array}{l}0.3(0.04- \\
2.5)\end{array}$} & \multirow[t]{3}{*}{0.475} \\
\hline & \multirow{2}{*}{$\begin{array}{l}37 \\
(19.5)\end{array}$} & \multirow{2}{*}{$\begin{array}{l}153 \\
(80.5)\end{array}$} & & \\
\hline & & & 1.0 & \\
\hline MPS & $2(5.7)$ & $33(94.3)$ & $0.2(0.1-1.0)$ & 0.031 \\
\hline Yes & \multirow[t]{2}{*}{$\begin{array}{l}36 \\
(21.3)\end{array}$} & $\begin{array}{l}133 \\
(78.7)\end{array}$ & \multirow[t]{2}{*}{1.0} & \\
\hline \multicolumn{2}{|l|}{ No } & & & \\
\hline Maternal anemia & $3(27.3)$ & $8(72.7)$ & \multirow{3}{*}{$\begin{array}{l}1.7(0.43- \\
6.7) \\
1.0\end{array}$} & \multirow[t]{3}{*}{0.433} \\
\hline Yes & \multirow{2}{*}{$\begin{array}{l}35 \\
(18.1)\end{array}$} & \multirow{2}{*}{$\begin{array}{l}158 \\
(819)\end{array}$} & & \\
\hline No & & & & \\
\hline Caesarean section delivery & $5(21.7)$ & $18(78.3)$ & \multirow{3}{*}{$\begin{array}{l}1.2(0.4- \\
3.6) \\
1.0\end{array}$} & \multirow[t]{3}{*}{0.776} \\
\hline Yes & \multirow{2}{*}{$\begin{array}{l}33 \\
(18.2)\end{array}$} & \multirow{2}{*}{$\begin{array}{l}148 \\
(81.8)\end{array}$} & & \\
\hline No & & & & \\
\hline Maternal Body Mass index (BMI) & $5(15.6)$ & $27(84.4)$ & \multirow{2}{*}{$\begin{array}{l}1.1(0.4- \\
3.2)\end{array}$} & 0.899 \\
\hline$<18.5 \mathrm{~kg} / \mathrm{m}^{2}$ (Underweight) & \multirow{2}{*}{$\begin{array}{l}15 \\
(14.7)\end{array}$} & 87 (85.3) & & 0.080 \\
\hline $18.5-25.0 \mathrm{~kg} / \mathrm{m}^{2}$ (normal) & & $39(73.6)$ & \multirow{2}{*}{$\begin{array}{l}1.0 \\
2.1(0.9- \\
4.7)\end{array}$} & \multirow[t]{2}{*}{0.363} \\
\hline $25.0-29.9 \mathrm{~kg} / \mathrm{m}^{2}$ (overweight) & $\begin{array}{l}14 \\
(26.4)\end{array}$ & 13 (76.5) & & \\
\hline > 30 kg/m² (obese) & \multicolumn{2}{|l|}{$4(23.5)$} & \multicolumn{2}{|l|}{$\begin{array}{l}1.8(0.5- \\
6.2)\end{array}$} \\
\hline $\begin{array}{l}\text { Any other maternal infections/conditions/co- } \\
\text { morbidities }\end{array}$ & $4(23.5)$ & $13(76.5)$ & $\begin{array}{l}1.4(0.4- \\
4.5)\end{array}$ & 0.531 \\
\hline Yes & $\begin{array}{l}34 \\
(18.3)\end{array}$ & $\begin{array}{l}152 \\
(81.7)\end{array}$ & 1.0 & \\
\hline No & & & & \\
\hline
\end{tabular}




\begin{tabular}{|c|c|c|c|c|}
\hline \multirow[t]{3}{*}{ Variable } & \multicolumn{2}{|l|}{ APFO } & \multirow[t]{3}{*}{ OR (95\% Cl) } & \multirow{3}{*}{$\begin{array}{l}P \\
\text { value }\end{array}$} \\
\hline & Yes & No & & \\
\hline & n (\%) & n (\%) & & \\
\hline Obstetrical Interventions & $2(33.3)$ & $4(66.7)$ & 1.0 & - \\
\hline \multirow{3}{*}{$\begin{array}{l}\text { Stress test } \\
\text { Amniocentesis } \\
\text { Tocolysis }\end{array}$} & $\begin{array}{l}27 \\
(18.8)\end{array}$ & $\begin{array}{l}117 \\
(81.3)\end{array}$ & $\begin{array}{l}0.5(0.08- \\
2.7)\end{array}$ & \multirow{3}{*}{$\begin{array}{l}0.386 \\
0.237\end{array}$} \\
\hline & \multirow{2}{*}{$1(9.1)$} & \multirow{2}{*}{10 (90.9) } & \multirow{2}{*}{$\begin{array}{l}0.2(0.01- \\
2.9)\end{array}$} & \\
\hline & & & & \\
\hline \multirow{3}{*}{$\begin{array}{l}\text { Positive } \\
\text { Negative }\end{array}$} & $\begin{array}{l}35 \\
(19.3)\end{array}$ & $\begin{array}{l}146 \\
(80.7)\end{array}$ & $\begin{array}{l}1.6(0.5- \\
5.7)\end{array}$ & 0.580 \\
\hline & \multirow{2}{*}{$3(13.0)$} & \multirow{2}{*}{$20(87.0)$} & \multirow{2}{*}{1.0} & \\
\hline & & & & \\
\hline Parity & \multirow{2}{*}{$\begin{array}{l}12 \\
(13.6)\end{array}$} & $76(86.4)$ & \multirow{3}{*}{$\begin{array}{l}1.0 \\
1.8(0.8- \\
3.9)\end{array}$} & - \\
\hline 1 & & $75(78.1)$ & & 0.149 \\
\hline $2-3$ & $\begin{array}{l}21 \\
(21.9)\end{array}$ & $13(72.2)$ & & 0.145 \\
\hline $4-5$ & $5(27.8)$ & $2(100)$ & \multirow{2}{*}{$\begin{array}{l}2.4(0.7- \\
8.1)\end{array}$} & \\
\hline $6-7$ & \multicolumn{2}{|l|}{0} & & \\
\hline Any previous adverse infant outcome & $6(25.0)$ & $18(75.0)$ & $\begin{array}{l}1.5(0.6- \\
4.2)\end{array}$ & \multirow[t]{3}{*}{0.406} \\
\hline Yes & \multirow{2}{*}{$\begin{array}{l}32 \\
(17.8)\end{array}$} & \multirow{2}{*}{$\begin{array}{l}148 \\
(82.2)\end{array}$} & \multirow{2}{*}{1.0} & \\
\hline No & & & & \\
\hline
\end{tabular}

\section{Multivariate analysis of risk predictors for adverse pregnancy-fetal outcomes:}

Multivariate analysis was performed to evaluate the independent risk factors associated with adverse pregnancy-fetal outcomes. Seven (7) variables that were associated with adverse pregnancy-fetal outcomes at $\mathrm{P}<0.05$ during bivariate analysis were considered in a multiple regression analysis. These included: (1) woman's IRIS status, (2) Separated marital status, (3) opportunistic infections, (4) HIV-RNA viral loads at baseline, (5) Cluster of differentiation at baseline,(6) Mothers general health during delivery and (7) maternal placental syndrome with an hypertensive event. After running all these factors using multinomial logistic regression by specifying 'backward conditional'progressive stepwise model with removal at $P<0.05$, three (3) factors were retained in the final analysis (reduced model) as presented in Table 4.4. Among women who experienced any form of adverse pregnancy outcome, there was almost 3 times more likely to have HIV-RNA viral load at baseline of above $50 \mathrm{copies} / \mathrm{ml}$ when compared to women who did not experience any form of adverse pregnancy outcome $[A O R=2.7 ; 95 \% \mathrm{Cl}: 1.2-6.3 ; \mathrm{P}$ $=.017]$. Maternal placental syndrome characterized by hypertensive event was not conclusive though at 
reduced model in terms of association with adverse pregnancy-fetal outcomes. This particular result is unlikely to have arisen purely by chance $[\mathrm{AOR}=0.1 ; 95 \% \mathrm{Cl}: 0.0-1.0 ; \mathrm{P}=.052]$. Mothers General health during delivery was about four (4) fold more likely to be among women experiencing adverse pregnancyfetal outcomes than women who did not experience adverse pregnancy outcome, $[\mathrm{AOR}=4 ; 95 \% \mathrm{Cl}$ : 4.0 : $1.8-9.1 ; P=.001]$. Surprisingly, all the other predictors at full and reduced model were found to be insignificant. Again, mother's Immune reconstitution inflammatory response syndrome status as the main predictor variable was not found to be associated with adverse pregnancy outcome $[\mathrm{AOR}=1.6 ; 95 \% \mathrm{Cl}$ : $0.4-5.8 ; P=.508]$ after controlling for significant moderating variables during multiple regression analysis (Table 6). 
Table 6

Multivariate analysis of risk factors associated with adverse pregnancy-fetal outcomes

\begin{tabular}{|lll|}
\hline Variable & AOR (95\% CI) & P value \\
\hline Full model & & \\
\hline IRIS & $1.6(0.4-5.8)$ & 0.508 \\
Yes & 1.0 & \\
No & & \\
\hline Marital status & $1.4(0.5-3.6)$ & 0.529 \\
Single & 1.0 & 0.259 \\
Married & $2.4(0.5-11.5)$ & 1.000 \\
Separated & - & \\
Widowed & & \\
\hline Opportunistic infections & $1.8(0.7-4.5)$ & 0.200 \\
Yes & 1.0 & \\
No & & \\
\hline CD4 counts at baseline & & \\
$<350$ & $0.5(0.1-1.8)$ & 0.293 \\
$>350$ & 1.0 & \\
\hline HIV viral load at baseline & & \\
$>$ 50 copies/ml & & \\
< 50 copies/ml & & \\
\hline MPS & $2.8(1.1-7.2)$ & 0.036 \\
Yes & 1.0 & \\
No & & \\
\hline Mothers General health during delivery & $3.2(1.3-7.9)$ & \\
Sick & 1.0 & \\
Not Sick & & \\
\hline Reduced model & & \\
\hline
\end{tabular}




\begin{tabular}{|lll|}
\hline Variable & AOR $(95 \% \mathrm{Cl})$ & P value \\
\hline HIV viral load at baseline & $2.7(1.2-6.3)$ & 0.017 \\
$>50$ copies $/ \mathrm{ml}$ & 1.0 & \\
$<50$ copies $/ \mathrm{ml}$ & & \\
MPS & $0.1(0.0-1.0)$ & 0.052 \\
Yes & 1.0 & \\
No & & \\
Mothers General health during delivery & $4.0(1.8-9.1)$ & 0.001 \\
Sick & 1.0 & \\
Not Sick & & \\
\hline
\end{tabular}

$\mathrm{OR}=$ Odds Ratio, $\mathrm{Cl}=$ Confidence Interval, *Significant $\mathrm{P} \leq 0.05$ level

\section{Discussion}

Maternal HIV infection has been associated with adverse pregnancy-fetal outcomes; however, there is paucity of data regarding effect of HAART due to immune reconstitution inflammatory response syndrome in pregnancy. While the benefits of HAART for prevention of mother-to-child transmission of HIV (PMTCT) are undisputed, there have been some concerns regarding its possible adverse effects on pregnancy outcomes (16). There is growing evidence in published literature suggesting that ART might be causing adverse birth outcomes among pregnant women in developing countries with frequently observed adverse birth outcomes including low birth weight (LBW), Preterm Birth (PB), and Small for Gestational Age (SGA) (17). HIV infected women in pregnancy are put at a higher risk of complications such as an intra-uterine growth restriction (38). Since HIV was found to exist as an immune suppressing virus in 1980s, it has impacted negatively to maternal health at the same time contributing to pregnancy related adverse effects. HIV infected pregnant women initiating ART has an increased risk of adverse pregnancy-fetal outcomes (18). Although, PMTCT program was incepted in 2006 to mitigate the effects of maternal HIV infection and to prevent vertical HIV transmission in Kenya, HIV-infection treatment has indirectly been associated with adverse pregnancy-fetal outcomes like; LBW, preterm birth and neonatal mortality. Moreover, the incident rates of composite adverse pregnancy-fetal outcomes including PT, LBW, and SGA infants are higher than those among HIV negative women (19).

This study has demonstrated that, being diagnosed with maternal HIV immune reconstitution inflammatory response syndrome increases the incidence rate and risk of adverse pregnancy outcome in the bivariate analysis among IRIS exposed as compared to the non-IRIS exposed HIV positive ART naïve women. It has been reported that adverse birth and pregnancy outcomes are linked to co morbidities 
among pregnant women receiving ART therapy by posing a danger to pregnancy and leading to ultimate adverse maternal, birth or pregnancy outcomes. However, after controlling for confounding factors, the association of maternal-HIV IRIS with adverse pregnancy-fetal outcomes was found not to be significant. Hence the finding of this prospective cohort study supports the null hypothesis which states that "there is no significant difference in the incidence of adverse pregnancy-fetal outcomes between women with maternal HIV-Immune Reconstitution Inflammatory Syndrome and women without maternal HIV-Immune Reconstitution Inflammatory Syndrome women attending selected referral facilities in Kenya". There is some evidence that HIV prevalence is higher among women as compared to men in Africa which is also replicated in Kenya (20). HIV-infection among pregnant women has been linked with multiple adverse pregnancy-fetal outcomes as compared to non-infected which was due to use of ART, as per the study done in China where adverse pregnancy-fetal outcomes were assessed by maternal HIV infection status and HIV-related factors using logistic regression analysis. The incidences of stillbirth (3.9\% vs $1.1 \%$ ), preterm birth (PTB) (8.9\% vs 3.7\%), low birth weight (LBW) $(12.2 \%$ vs $3.1 \%)$ and small for gestational age (SGA) $(21.3 \%$ vs $7.0 \%)$ were higher in HIV-infected women than HIVuninfected women, with adjusted ORs of 2.77 (95\% Cl: $1.24-6.17), 2.37$ (95\% Cl: $1.44-3.89), 4.20$ (95\% Cl: $2.59-6.82)$ and $3.26(95 \% \mathrm{Cl}: 3.26-4.64)$, respectively (21)

A Systematic Review study conducted by Fekadu et al in 2015 showed that, there is growing evidence in published literature suggesting that ART might be causing adverse birth outcomes among pregnant women in developing countries, an issue which still was not established if it was the immune responses following the ART therapy with the debate as to the role of maternal HAART as a risk factor for adverse pregnancy outcome having been on discussion (22). Subsequently, association between maternal ART in HIV among pregnant women and pregnancy outcomes in a systematic review study on use of antiretroviral agents in pregnant HIV-infected women has been reported to increase the risk of premature delivery (23). In another study done in India, HIV-infected women were more likely to have PTB, IUGR, and anemia $(9.4 \%, 9.9 \%, 5.2 \%)$ compared to uninfected women $(7.6 \%, 5 \%, 3.8 \%)$, this did not reach statistical significance $(P$-value $=>0.05)$. Neonatal intensive care unit admissions were also significantly higher in infants born to HIV-infected women with preceding opportunistic infections following ART therapy(Pvalue $=0.0020$ (24). Similarly, a study on the association between maternal HIV among ART treated women and perinatal outcome including neonatal mortality showed some positive association $(\mathrm{OR}=1.1$, $95 \%$ ci $0.63-1.93)(25)$.

In another study, among women initiating ART in pregnancy, HAART use was associated with higher odds of preterm delivery (AOR, 1.4; $95 \% \mathrm{Cl}, 1.2,1.8)$, SGA (AOR, 1.5; 95\% Cl, 1.2, 1.9), and SB (AOR, 2.5; $95 \% \mathrm{Cl}$, $1.6,3.9)(38)$. However, these studies are not related to this current prospective cohort study as they were carried out either on the direct relation of HIV infection adverse pregnancy-fetal outcomes as well as HAART in relation to adverse pregnancy or birth outcomes without focusing on the immune reconstitution inflammatory response syndrome which is purported in this study. Again they were conducted some in diverse populations without focusing on pregnant women, some were also on pregnant women who had already been initiated on ART therapy, while others concentrated possibly on ART combination and possible adverse drug effects on pregnancy without looking in to the salient issue behind immune 
reconstruction following ART therapy. Adverse pregnancy-fetal outcomes have, however, been reported more commonly in a number of African studies including complications of both early and late pregnancy. HIV may be the direct cause or a marker of a complex interaction of related medical and social conditions that affect pregnancy (26). Moreover, there are no studies conducted on the indirect effect of ART institution among ART naïve pregnant women on the immune reconstitution response and its possible predictor of adverse pregnancy-fetal outcomes. Again, this prospective study looked at a baseline risk of adverse pregnancy-fetal outcomes as HIV infection and only focused on the exposure of interest for adverse pregnancy outcome as maternal HIV- IRIS, a syndrome which may not be addressed during pregnancy yet it may have negative impacts on pregnancy outcome.

The research findings support the fact that, other factors other than maternal HIV-IRIS predict the adverse pregnancy-fetal outcomes. Maternal health during pregnancy which may be due to IRIS is associated with adverse pregnancy-fetal outcomes. Excess adverse pregnancy-fetal outcomes in HIV-infected ART naïve pregnant women is not primarily explained by the associated opportunistic infections due to IRIS but was strongly associated with HIV-RNA-loads of $>50 \mathrm{copies} / \mathrm{ml}$ at baseline, and possibly, any form of hypertensive disorder during pregnancy or maternal placental syndrome, similar to a study done in USA and Haiti (39); (27) respectively.

Maternal HIV infection has been noted to be associated with increased risks of stillbirth, PTB, LBW and SGA, even on the condition that most HIV-infected pregnant women are usually started on ARV therapy following a positive test for HIV (21). HIV infection among pregnant women has a peculiar clinical and immunological scenario due to the immunological changes meant to cater for the developing fetus, as well as the possible pharmacological effects of the HAART medication. The combination of these two factors seemingly complicates the gestational journey as compared to women who are HIV negative and not on any ART medication (28). There is also a possibility of HIV transmission from mother to child during pregnancy, labor, delivery or breastfeeding (perinatal transmission). Perinatal HIV transmission is the most common way children are infected with HIV with possible consequential adverse pregnancy and related birth outcomes (29).

Presence of any hypertensive disorder during pregnancy, although being protective at reduced model for adverse pregnancy-fetal outcomes, it was at the borderline and seemingly predicted the outcome but not conclusive as a risk factor associated with adverse pregnancy-fetal outcomes (AOR $=0.1,95 \% \mathrm{Cl}$ : $(0.0-$ 1.0); $P=.052$ ). Similar to this finding, in a systematic review and meta-analysis study, no single test of hypertensive disorder was a strong independent predictor of an adverse pregnancy outcome. The most promising prediction was with multivariable models, especially when oxygen saturation, or chest pain/dyspnea were included, just at borderline showing other inter-related clinical courses (30). In another cohort study, there was a mixed prediction of both protective and significant association for adverse pregnancy and birth outcomes: decreased risk of LGA (OR 0.65, 0.51-0.83), with a recommendation that; hypertensive disorder should be combined with other maternal characteristics, medical and obstetric history when calculating an individualized adjusted risk for adverse pregnancy complications. It was found that, hypertensive disorder increases the risk for stillbirth, PE, SGA, GDM, iatrogenic PTB and 
elective CS and reduces the risk for LGA (31). A study done in a facility based setting in China predicted adverse birth outcomes in a similar manner, precisely still birth which, the risk decreased as gestational age increased for all women and for all subtypes of hypertensive disorder and it was similar in hypertensive and normo-tensive women younger than 20 years of age. Among women with a hypertensive disorder in pregnancy, the stillbirth rate was strongly influenced by socio-demographic characteristics. Moreover, a stillbirth was more likely if the woman had received few antenatal care visits, was poorly educated, was single, widowed or divorced, had a vaginal delivery, had high parity or was older than 40 years (32). Likewise, a study conducted on 9133 singleton nulliparous pregnancies, neither blood pressure nor blood pressure and proteinuria are accurate predictors of severe adverse maternal and peri-natal outcomes with protective but borderline effect $(P=0.053)$.

Woman's general health during pregnancy and at delivery is a very key area of concern on the birth outcome. Mother's general health was highly significant for adverse pregnancy-fetal outcomes in this prospective cohort study. There are mechanisms thought to account for the synergy between neonatal mortality outcome and infections during pregnancy and delivery that lead to neonatal mortality by affecting proper fetal growth and development. Regarding this, women who were defined 'sick' case in this study had four times incident of adverse pregnancy-fetal outcomes compared to women who were not defined as sick within the same periods of time[OR $=4 ; 95 \% \mathrm{Cl}: 1.8-9.1 ; \mathrm{P}=.001]$, which is reflective to a case control study done in Jimma University on determinants of adverse pregnancy-fetal outcomes where; mothers who had illness during current pregnancy had seven times to be experience an adverse birth outcome than those who were nit ill, AOR $=7.22,95 \% \mathrm{Cl}: 1.65-31.58]$ (33). Another similar trend with Detection of $A$ (H1N1) pdm09-specific antibodies was associated with a lower 10th percentile of birth weight, $\beta=-159 \mathrm{~g}(95 \% \mathrm{Cl}-309,-9)$, with influenza infection during pregnancy predicting reduction of only but the birth weight of the smallest children (34). In another Chinese hospital based study, maternal HBsAg carriage was associated with increased risk of pregnancy-induced hypertension [adjusted odds ratio $(\mathrm{aOR})=2.20 ; 95 \%$ confidence interval $(\mathrm{Cl}), 1.30-3.73]$, fetal distress $(\mathrm{aOR}=1.40 ; 95 \% \mathrm{Cl}, 1.09-1.78)$, cesarean delivery $(\mathrm{aOR}=1.70 ; 95 \% \mathrm{Cl}, 1.45-1.99)$, and macrosomia $(\mathrm{aOR}=1.68 ; 95 \% \mathrm{Cl}, 1.19-2.37)(35)$. This finding compare well with study examining maternal characteristics associated with adverse pregnancy-fetal outcomes of a carried out by (19). Similarly, a study under the bulletin of WHO showed a pooled estimates of neonatal mortality were $12.3 \%(95 \% \mathrm{Cl}$ : 9.3-16.2) among women with syphilis and $3.0 \%(95 \% \mathrm{Cl}: 2.1-4.3)$ among women without, for an absolute difference of $9.3 \%$ showing more risk among women with syphilis and untreated (36).

HIV-RNA viral load of $>50$ copies/ml at the baseline during the first trimester among the HIV positive ART naïve women was found to be associated almost three times with adverse pregnancy-fetal outcomes as compared to HIV-RNA viral load of $<50$ copies/ml. This was in regard to being a risk factor for IRIS identification which was the main predictor variable for adverse pregnancy-fetal outcomes. This correlates with another USA based study on burden of viral load which showed that, extent of HIV replication during pregnancy, as represented by plasma HIV RNA viral load, predicted an adverse pregnancy outcome; the risk of pregnancy loss for those with $\log _{10}$ viral load $>4.00$ before pregnancy 
ended was 1.59 (95\% confidence interval $(\mathrm{Cl}): 0.99,2.56)$ times as high as the risk for women whose $\log _{10}$ viral load was $\leq 1$ (28). In a study, which although compared HIV positive women and HIV negative women, there was a significantly higher risk of low birth weight (RR 2.29,95\% Cl 1.34-3.92; $P=0.03$ ) and prematurity (<37 weeks) (RR $1.93,95 \% \mathrm{Cl} 1.35-2.77 ; P=0.0003$ ) among symptomatic HIV-infected women when compared with HIV-uninfected women. This symptomatic status in HIV is associated with higher viral load as compared to asymptomatic. It concluded that, HIV-infected women, particularly those who are symptomatic, are at a higher risk of adverse pregnancy-fetal outcomes (37) and (25).

The study indicates that adverse pregnancy-fetal outcomes were significantly associated with opportunistic infections $\mathrm{OR}=2.3 ; 95 \% \mathrm{Cl}:(1.1-4.8) ; \mathrm{P}=.013)$ in bivariate analysis, but it did not predict neonatal mortality outcome in multivariate level. This imitates a conference paper by Charlene and Megan, 2017. This fact of opportunistic infections predicting adverse pregnancy-fetal outcomes is commensurate with mothers general well being at delivery in this study which shows a four-fold prediction rate for adverse pregnancy-fetal outcomes at logistic regression, reduced model.

\section{Conclusions}

Prevention of MTCT was incepted in 2006 and the milestone so far has shown a great improvement. With increased roll-out of ART among HIV positive pregnant women, associated poor birth outcomes due to vertical HIV transmission have drastically reduced. The conclusions of this study are based on the findings and discussions regarding the effect of immune reconstitution syndrome on pregnancy outcome among HIV infected women starting ART in public hospitals in Nairobi, Kenya. The study indicates an indirect effect of ART, that is, the HIV associated immune reconstitution inflammatory response syndrome on pregnancy outcomes. This when timely addressed, the associated predictors of APFOs may be controlled and further reduce adverse pregnancy-fetal outcomes such as; low birth weight, preterm birth, neonatal sepsis and mortalities among others.

\section{Abbreviations}

AIDS cquired Immunodeficiency Syndrome

APGAR Appearance, Pulse, Grimace, Activity, and Respiration

APFOs Adverse pregnancy-fetal outcomes

aRR Adjusted Relative Risk

ART Anti-Retroviral Therapy

C.I Confidence Interval

CDC Centre for Disease Control 
CMV Cytomegalo-Virus

EDD Expected Delivery Date

ERC Ethical Review Committee

HAART Highly Active Antiretroviral Therapy

HICs High Income Contries

HIV Human Immunodeficiency Virus

IRIS Immune Reconstitution Inflammatory Syndrome

KDHS Kenya Demographic and Health Survey

LBW Low Birth Weight

LMICs Lower Middle Income Contries

$\mathrm{MOH}$ Ministry of Health

MPS Maternal Placental Syndrome

MTCT Mother to child transmission

MTCT Mother to Child Transmission

O.Is Opportunistic Infections

OR Odds Ratio

PTB Preterm Birth

RR Relative Risk

SB Still Birth

SDGs Sustainable Development Goals

SGA Small for Gestational Age

SPSS Statistical Package for Social Sciences

SSA Sub-Saharan Africa

STD Sexually Transmitted Diseases 
STI Sexually Transmitted Infection

TB Tuberculosis

UN United Nations

UNAIDS United Nations Program on HIV/AIDS

UNFPA United Nations Populations Fund

UNICEF United Nations International Children's Emergency Fund

URR Unadjusted Relative Risk

USA United States of America

VCT Voluntary Counseling and Testing

WHO World Health Organization

\section{Declarations}

\section{Ethics approval and consent to participate}

This study was reviewed and approved by the University of Nairobi-Kenyatta National Hospital Ethical Review Committee (reference number: P-609/08/2018). All participants provided written informed consent, and the ethics committee approved the procedures.

\section{Consent for publication}

Not applicable

\section{Availability of data and materials}

The datasets used and/or analyzed during the current study are available from the corresponding author on reasonable request.

\section{Competing Interests}

The authors declare that they have no competing interests

\section{Authors' contributions}

JM played a major role in conception of the study, design, played key role in data analysis and drafting of the work. YK played a role in revision and interpreted the patient data regarding the immune reconstitution syndrome. AM was a contributor in writing the final draft, guidance and interpretation of 
general data. MK was involved in drafting the work with a substantive revision and a role in manuscript development and guidance. All authors have read and approved the final version of the manuscript.

\section{Acknowledgements}

We truly appreciate all the researchers' commitment in data collection. We acknowledge and thank Mbagathi and Kenyatta National Hospitals for allowing the study to be carried in the facilities. We are in addition, indebted to all of the study participants, the ART naïve pregnant women in this prospective cohort study. We appreciate the immense expertise by the HIV specialists in diagnosing the maternal HIV $-I R I S$.

\section{Funding}

The author received no funding for this work

\section{References}

1. INSIGHT START Study Group. Initiation of antiretroviral therapy in early asymptomatic HIV infection.N Engl J Med2015

2. Naomi F Walker, James Scriven, Graeme Meintjes, Robert J Wilkinson. Immune reconstitution inflammatory syndrome in HIV-infected patients .HIV AIDS (Auckl). 2015; 7: 49-64. Published online 2015 Feb 12. doi: 10.2147/HIV.S42328PMCID: PMC4334287

3. Mitroi-Maxim, C., Dumea, E., Halichidis, S. et al. Opportunistic infections and immune reconstitution inflammatory syndrome (IRIS) in HIV infected patient - late presenter in cART era: case report. BMC Infect Dis 14, P43 (2014). https://doi.org/10.1186/1471-2334-14-S4-P43

4. Kaguthi Grace, Videlis Nduba, Martien Wilhelm Borgdorff, Suzanne Verver. Predictors of post neonatal mortality in Western Kenya: a cohort studyPan Afr Med J. 2018; 31: 114. Published online 2018 Oct 15. doi: 10.11604/pamj.2018.31.114.16725PMCID: PMC6462392

5. Naomi F. Walker, Cari Stek, Sean Wasserman, Robert J. Wilkinson, Graeme MeintjesThe tuberculosisassociated immune reconstitution inflammatory syndrome: recent advances in clinical and pathogenesis research Curr Opin HIV AIDS. 2018 Nov; 13(6): 512-521.

6. Sereti, Virginia Sheikh, Douglas Shaffer, Nittaya Phanuphak, Erin Gabriel, Jing Wang, Martha C Nason, Gregg Roby, Hellen Ngeno, Fredrick Kirui, Alice Pau, Joann M Mican, Adam Rupert, Rachel Bishop, Brian Agan, Nitiya Chomchey, Nipat Teeratakulpisarn, Somsit Tansuphaswadikul, Deborah Langat, Josphat Kosgei, Martyn French, Jintanat Ananworanich, Fredrick Sawe, Prospective International Study of Incidence and Predictors of Immune Reconstitution Inflammatory Syndrome and Death in People Living With Human Immunodeficiency Virus and Severe Lymphopenia, Clinical Infectious Diseases, Volume 71, Issue 3, 1 August 2020, Pages 652-660

7. Sharma LK, Dutta D, et al.: Prevalence and predictors of thyroid dysfunction in patients with HIV infection and acquired immunodeficiency syndrome: An Indian perspective. J Thyroid Res 2015; 
2015: 517173.

8. French MA. HIV/AIDS: immune reconstitution inflammatory syndrome: a reappraisal. Clin Infect Dis 2015; 48:101.

9. David B Meya, Yukari C Manabe, David R Boulware, Edward N Janoff. The Immunopathogenesis of Cryptococcal Immune Reconstitution Inflammatory Syndrome - Understanding a ConundrumCurr Opin Infect Dis. Author manuscript; available in PMC 2017 Feb 1.Published in final edited form as: Curr Opin Infect Dis. 2016 Feb; 29(1): 10-22.

10. Narendran, G., Oliveira-de-Souza, D., Vinhaes, C.L. Multifocal tuberculosis-associated immune reconstitution inflammatory syndrome - a case report of a complicated scenario. BMC Infect Dis 19, 529 (2019)

11. Stacey A. Hurst, Kristie E. Appelgren, Athena P. Kourtis. Prevention of mother-to-child transmission of Human Immunodeficiency Virus Type 1 (HIV): the role of neonatal and infant prophylaxisExpert Rev Anti Infect Ther. Author manuscript; available in PMC 2016 Feb 1.Published in final edited form as: Expert Rev Anti Infect Ther. 2015 Feb; 13(2): 169-181

12. Narendran Ornella, Seipati Mothebesoane-Anoh, Patricia Gomez, Stephen Munjanja. The role of the community: Antenatal Care - World Health Organization

13. Saag MS, Benson CA, Gandhi RT, et al. Antiretroviral drugs for treatment and prevention of HIV infection in adults: 2018 recommendations of the International Antiviral Society-USA panel. JAMA. 2018;320(4):379-396.

14. María Pérez- Narendran , Michele Hernández-Cabrera, Adela Francés-Urmeneta, Alfonso AngelMoreno, Elena Pisos-Álamo, Nieves Jaén-Sánchez, Cristina Carranza-Rodríguez, Jose-Luis PérezArellano. Immune Reconstitution Inflammatory Syndrome in HIV-Infected Immigrants. The American Journal of Tropical Medicine and Hygiene, Volume 97, Issue 4, 11 Oct 2017, p. 1072 - 1077

15. Madhu Chhanda Choudhary. Antiretroviral Therapy (ART) in Pregnant Women With HIV Infection Overview of HIV Antiretroviral Therapy (ART) in PregnancyUpdated: Jun 23, 2020

16. Short CE, Douglas M, Smith JH, et al. . Preterm delivery risk in women initiating antiretroviral therapy to prevent HIV mother-to-child transmission. HIV Med 2014-2015;15:233-8. 10.1111/hiv.1208

17. Fekadu Mazengia Alemu, Alemayehu Worku Yalew, Mesganaw Fantahun, Eta Ebasi Ashu. Antiretroviral Therapy and Pregnancy Outcomes in Developing Countries: A Systematic Reviewlnt J MCH AIDS. 2015; 3(1): 31-43. PMCID: PMC4948169

18. Stringer, Elizabeth M. ,Michelle A. Kendall, Shahin Lockman, Thomas B. Campbell, Karin NielsenSaines, Fred Sawe, Susan Cu-uvin, Xingye Wu, Judith S. Currier. Pregnancy outcomes among HIVinfected women who conceived on antiretroviral therapy PLoS One. 2018; 13(7)

19. Regis Kreitchmann, Su X. Li, Victor Hugo Melo, Debora Fernandes Coelho, D. Heather Watts, Esau Joao, Conrado Milani Coutinho, Jorge O. Alarcon,George K. Siberry. Predictors of Adverse pregnancyfetal outcomes in HIV infected Women in Latin America and the Caribbean: a Cohort StudyBJOG. Author manuscript; available in PMC 2015 Nov 1.Published in final edited form as: BJOG. 2014 Nov; 
121(12): 1501-1508. Published online 2014 Mar 7. doi: 10.1111/1471-0528.12680PMCID:

PMC4157114

20. KDHS 2016-The 2016 Kenya Demographic and Health Survey (2016KDHS) of October

21. Huixia Li., Liu, J., Tan, D., Huang, G., Zheng, J., Xiao, J., Wang, H., Huang, Q., Feng, N., \& Zhang, G. (2020). Maternal HIV infection and risk of adverse pregnancy-fetal outcomes in Hunan province,China:A prospective cohortstudy.Medicine,99(8),e19213.https://doi.org/10.1097/MD.0000000000019213

22. Bisio F , Nicco E, Calzi A, et al . Pregnancy outcomes following exposure to efavirenz-based antiretroviral therapy in the Republic of Congo. New Microbiol 2015;38:185-92

23. Yohannes Ejigu,Jeanette H Magnus, Johanne Sundby, and Maria C Magnus. Pregnancy outcome among HIV-infected women on different antiretroviral therapies in Ethiopia: a cohort study2019; 9(8): e027344. Published online 2019 Aug5.doi: 10.1136/bmjopen-2018-027344PMCID:

PMC6687026PMID: 31383698

24. Vatsla Dadhwal, Aparna Sharma, Kavita Khoiwal, Dipika Deka, Plaboni Sarkar, P. Vanamail. Pregnancy Outcomes in HIV-Infected Women: Experience from a Tertiary Care Center in Indialnt $\mathrm{J}$ MCH AIDS. 2017; 6(1): 75-81. doi: 10.21106/ijma.196PMCID: PMC5547228

25. Xiao, P., Zhou, Y., Chen, Y. et al. Association between maternal HIV infection and low birth weight and prematurity: a meta-analysis of cohort studies. BMC Pregnancy Childbirth 15, 246 (2015). https://doi.org/10.1186/s12884-015-0684-z x

26. Sebitloane, HM., J Moodley. Maternal and obstetric complications among HIV-infected women treated with highly active antiretroviral treatment at a Regional Hospital in Durban, South Africa: Nigerian Journal of Clinical Practice, 2017, Volume: 20 Issue: 11 Page: 1360-1367

27. Bridwell, M., Handzel, E., Hynes, M. et al. Hypertensive disorders in pregnancy and maternal and neonatal outcomes in Haiti: the importance of surveillance and data collection. BMC Pregnancy and Childbirth 19, 208 (2019). https://doi.org/10.1186/s12884-019-2361-0

28. Jean de Dieu Anoubissi , Ekali Loni Gabriel, Cyprien Kengne Nde, Joseph Fokam, Dorine Godelive Tseuko, Arlette Messeh, Yasmine Moussa, Celine Nguefeu Nkenfou, Leonard Bonono, Serge-Clotaire Billong, Jean-Bosco Elat Nfetam . Factors associated with risk of HIV-infection among pregnant women in Cameroon: Evidence from the 2016 national sentinel surveillance survey of HIV and syphilis. PLoS One. 2019; 14(4): e0208963. Published online 2019 Apr 12. doi: 10.1371/journal.pone.0208963

29. World Health Organization Preventing Mother-to-Child Transmission of HIV CDC's HIV Basics;Date last updated: December 19, 2018

30. Von Dadelszen, Peter, and Laura A Magee. "Preventing deaths due to the hypertensive disorders of pregnancy." Best practice \& research. Clinica/ Best Pract Res Clin Obstet Gynaecol. 2016 Oct; 36: 83102. doi: 10.1016/j.bpobgyn.2016.05.005

31. Harmsen, M.J., Browne, J.L., Venter, F. et al. The association between HIV (treatment), pregnancy serum lipid concentrations and pregnancy outcomes: a systematic review. BMC Infect Dis 17, 489 
(2017). https://doi.org/10.1186/s12879-017-258

32. Xiong, Yi Mu, Juan Liang, Jun Zhu, Xiaohong Li, Jinke Li, Zheng Liu, Yi Qu, Yanping Wang, Dezhi MuHypertensive disorders in pregnancy and stillbirth rates: a facility-based study in ChinaBull World Health Organ. 2018 Aug 1; 96(8): 531-539. Published online 2018 Jun 12. doi: 10.2471/BLT.18.208447PMCID: PMC6083384

33. Eyosiyas Yeshialem, Nigus Alemnew, Mulumebet Abera and Amanuel Tesfay. Determinants of Adverse pregnancy-fetal outcomes among mothers who gave birth from Jan 1-Dec 31/2015 in Jimma University Specialized Hospital, Case control study, 2016. Journal of public health in developing contries, DOI: 10.21767/2471-299X.1000063

34. IdaLaake, Gro Tunheim, Anna Hayman Robertson, Olav Hungnes, Kristian Waalen, Siri E. Håberg,Siri Mjaaland\&Lill Trogstad. Risk of pregnancy complications and adverse birth outcomes after maternal A(H1N1)pdm09 influenza: a Norwegian population-based cohort study BMC Infectious Diseasesvolume18, Article number: 525 (2018) Cite this article

35. Wan, Zhihua MD*; Zhou, Aifen MD+; Zhu, Huiping MD; Lin, Xiaofang MD*; Hu, Dan MD*; Peng, Songxu MD*; Zhang, Bin MD; Du, Yukai MM*. Maternal Hepatitis B Virus Infection and Pregnancy Outcomes. A Hospital-based Case-control Study in Wuhan, China. Journal of Clinical Gastroenterology: January 2018 - Volume 52 - Issue 1 - p 73-

78doi:10.1097/MCG.0000000000000842

36. Eline L. Korenromp, Jane Rowley, Monica Alonso, Maeve B. Mello, N. Saman Wijesooriya, S. Guy Mahiané, Naoko Ishikawa, Linh-Vi Le, Morkor Newman-Owiredu, Nico Nagelkerke, Lori Newman, Mary Kamb, Nathalie Broutet, Melanie M. Taylor. Global burden of maternal and congenital syphilis and associated adverse birth outcomes-Estimates for 2016 and progress since 2012

37. Jenny L. Coley, Gernard I. Msamanga, Mary C. Smith Fawzi, Sylvia Kaaya, Ellen Hertzmark, Saidi Kapiga, Donna Spiegelman, David Hunter, Wafaie W. FawziThe association between maternal HIV-1 infection and pregnancy outcomes in Dar es Salaam, TanzaniaBJOG. Author manuscript; available in PMC 2018 Dec 3.BJOG. 2001 Nov; 108(11): 1125-1133. PMCID: PMC6276363

38. Arab, K., Spence, A.R., Czuzoj-Shulman, N. et al. Pregnancy outcomes in HIV-positive women: a retrospective cohort study. Arch Gynecol Obstet 295, 599-606 (2017).

\section{Figures}




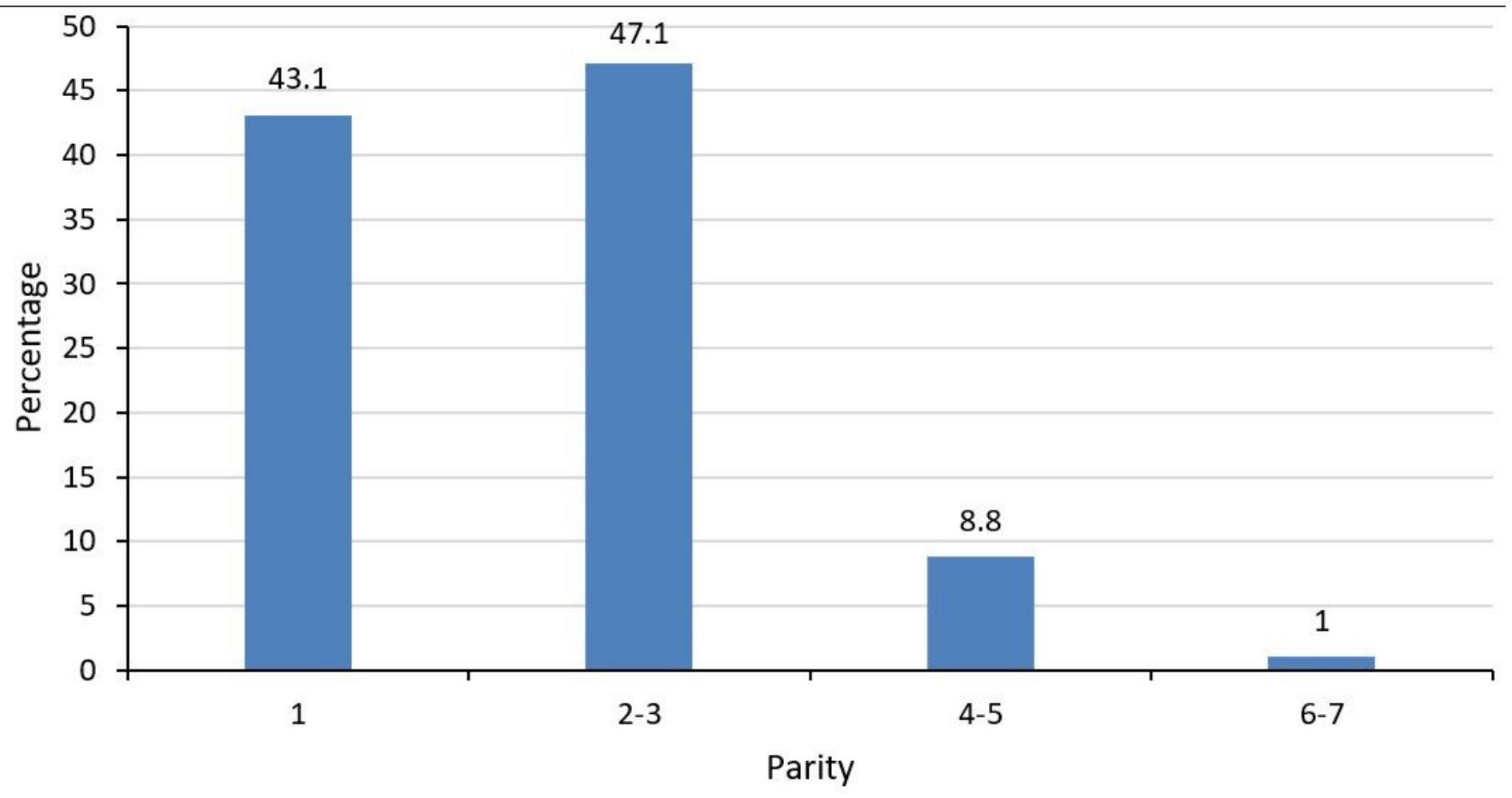

Figure 1

Parity distributions among all women

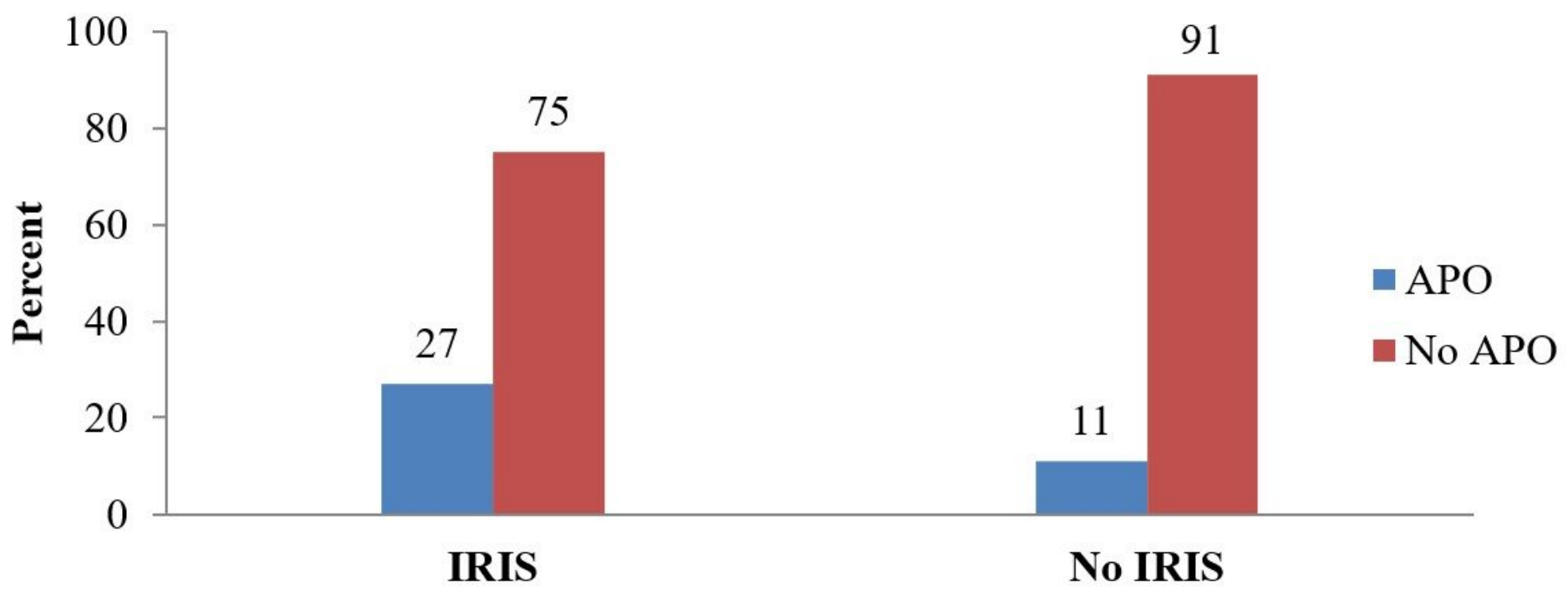

Figure 2

Distribution of adverse pregnancy-fetal outcomes for women with and women without maternal HIV immune reconstitution inflammatory response syndrome 